\title{
Novel reusable transosseous tunnel based soft tissue repair techniques about the shoulder: a rational, value based approach
}

Volume 5 Issue I - 2016

\section{Conceptual Paper}

The value based approach to medical care has created a new practice environment which is more focused on delivering noninferior or equivalent evidence based outcomes at a sustainable cost. In a system where value based principles have been slowly adopted, shoulder care is ideally suited to provide leadership in value based medical practice. ${ }^{1}$ Technology remains a high cost driver for health systems, and surgeon owned facilities or gain sharing arrangements are beginning to enable the opportunity to co-align technology end users with payor's incentives and that of institutional value assessment committees, which is critical to foster adoption of a value based approach. ${ }^{2}$ With regard to rotator cuff repair, outpatient rotator cuff repair saves cost when compared to inpatient repair, and open cuff repair is more cost effective compared to all arthroscopic. ${ }^{3,4}$ There is also evidence that anchor burden may increase cost significantly. ${ }^{5,6}$ Adla et al. ${ }^{7}$ showed that arthroscopic cuff repair was less cost effective by an average of $\$ 1,248.75$ per case. ${ }^{7}$ Narvy et al. ${ }^{8}$ recently defined the direct economic cost of rotator cuff repair in the outpatient setting as $\$ 5,904.21 .{ }^{8}$ In their study, implantable anchors were the dominant cost driver, with a mean anchor cost per case of $\$ 3,432.67$, with double row repairs averaging $\$ 4,570.25$ anchor cost per case. In a similar vein, Genuario concluded in a decision-analytic model that double row rotator cuff repair was not cost effective for any size tear. ${ }^{5}$ While there is evidence that anchors increase cost, there is little evidence that any one implant or method is superior to another in terms of implants or repair techniques. ${ }^{9,10}$ Because clinical outcome does not correlate well with structural integrity of the cuff after repair, it is likely that various implants and techniques will be equivalent or non-inferior in evidence based analysis. ${ }^{11}$ Arthroscopic soft tissue repair about the shoulder offers a prime opportunity to provide high value, time honored repair techniques with updated cost effective arthroscopic approaches for our changing environment.

Transosseous cuff repair has been utilized with good success for decades, and is considered by many to be the gold standard. ${ }^{12-15}$ Arthroscopic transosseous approaches for cuff repair have been previously described in literature. ${ }^{16-20}$ More recently, arthroscopic tunneling has been performed with comparable results to standard anchor approaches ${ }^{21-24}$ In a recent retrospective case-control study of anchor based versus anchorless transosseous repairs, Srikumaran et al. ${ }^{6}$ showed no difference in ASES scores, NRS pain scores, SSV, procedural time, or ultrasound proven tendon integrity between arthroscopic transosseous technique and anchor based repairs for rotator cuff repair. ${ }^{6}$ Despite encouraging early evidence, arthroscopic transosseous technique has not been widely adopted, possibly because of technical difficulties, lack of familiarity, lack of marketing, or cost of a disposable device. To mitigate some of these concerns, a simple, reliable, and reusable device has been designed to enable all arthroscopic or open approaches to soft tissue repair in the outpatient

\author{
Brett Sanders \\ Center for Sports Medicine, USA
}

Correspondence: Brett Sanders, Center for Sports Medicine, 26 ridge rock drive signal mountain, TN 37377, USA, Email sandbrett@gmail.com

Received: May 02, 2016 | Published: May 12, 2016

setting for shoulder and other soft tissue repair procedures that may offer viable cost effective approaches with limited technology cost burden in a more sustainable fashion. As bundling payment methodologies approach, surgeons may be more incentivized to consider cost in the episode of care. This article will explore novel all transosseous and hybrid arthroscopic techniques and rational for soft tissue repair about the shoulder.

The Transos tunneler (tensor surgical, chattanooga, TN) is a reusable, value-based device which enables accurate and reliable tunneling through the humerus with simple retrieval mechanisms and simultaneous suture delivery and retrieval, reducing the number of steps and complexity in creating tunnels arthroscopically. (Figure 1) Similarly, it passes suture through compacted rather than drilled bone tunnels for added mechanical strength. This device can be used as a stand-alone device or in combination with anchors for a hybrid approach to soft tissue repair. Reusable tunnel creation allows extra fixation points and circumferential compression at no additional cost, thereby maximizing outcome and minimizing cost.

All transosseous approaches to rotator cuff repair have been previously described in the early 20th century, and are considered by many to still be the gold standard repair. Current technology allows the arthroscopist to create simple suture repairs, box repairs, or $\mathrm{x}$ box repairs with mattress and crossing sutures. ${ }^{18,19,25}$ Biomechanical studies have shown similar properties in anchor and tunnel repairs, ${ }^{25,26}$ with higher technology cost for the tunnel repairs. ${ }^{21}$ Early clinical studies have shown favorable results when comparing anchor based and arthroscopic tunnel repairs. ${ }^{22,23}$ Anchorless repairs may offer some potential advantages over anchor based repairs, given that Johst et al. ${ }^{27}$ have shown that strength or repair is determined only by the number of sutures crossing the repair site, not necessarily the number of anchors used. ${ }^{27}$

Biological considerations are currently believed to be the predominant factor in rotator cuff healing, along with clinical factors such as timing of repair, host factors, size of tear, and chronicity. 
An excellent biological milieu is created in transosseous repairs by allowing easy ingress of stem cells from the proximal humerus bone marrow to the repair site with no inert substance in the tunnel to block access. Bone tunnels essentially mimic the micro fracture technique of the tuberosity which has been linked with increased healing of the rotator cuff. ${ }^{28,29}$ The Transos device creates a $2.9 \mathrm{~mm}$ medial and $1.9 \mathrm{~mm}$ lateral tunnel, so there are no problematic bone voids in revision cases, and an anchor can always be introduced into the small diameter hole in the case of soft bone, a bone cyst, or any technical problem. Failure mode of transosseous repairs typically spare tendon substance, thus creating a less complex revision surgery in the case of a re-tear. If the failure mode occurs through suture cut through in the bone, it has occurred through one of the only tissues in the body that can heal with regenerative healing, allowing more options for revision fixation.
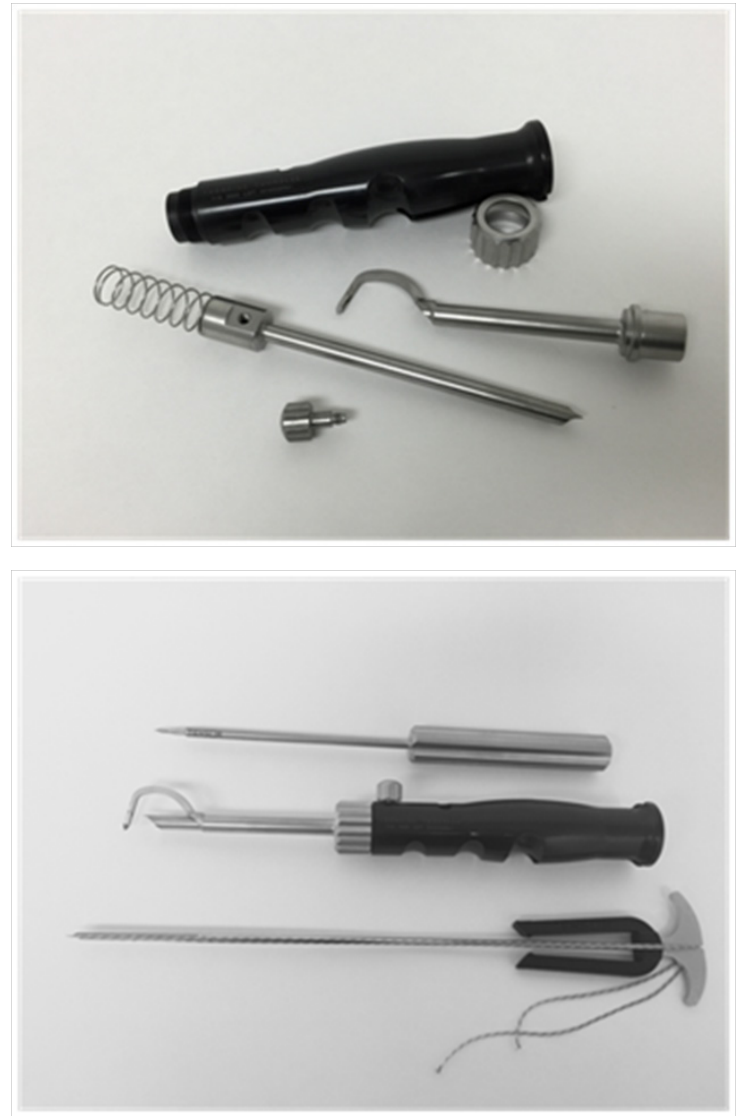

Figure I A reusable transosseous tunneler design breaks down for sterilization and allows repeatable transosseous tunneling within the case.

If the failure mode of the repair construct is shifted to the biologically disadvantaged rotator cuff tendon, tendon truncation may occur, requiring a graft for salvage. Some anchor constructs exceeding the tendon modulus may create medial stress risers and tissue strangulation, ${ }^{30}$ which could contribute to tendon substance loss and difficult revision circumstances. Additional technical benefits of transosseous tunnels include excellent footprint area restoration through circumferential tendon compression, the ability to make large bone bridges with mattress sutures while simultaneously sealing off fibrinolytic synovial fluid from the repair site, and two fixation points per tunnel. Additionally, the surgeon may choose the number of sutures placed in the tunnel for added strength, and create unlimited repeat tunnels within a case with no extra cost burden. This is an important facet when considering that strength of the composite repair parallels the number of sutures through the tendon, not the strength of a boneanchor interface. ${ }^{27}$ Similiarly, more tunnel fixation points with more sutures gives the surgeon an option for increasingly strong repair with a higher cost ceiling compared to increasing anchor fixation points alone. Arthroscopic grasping sutures and suture tapes are further methods to add strength to tunneled repair constructs. It is possible that a new goal in arthroscopic rotator cuff surgery should not be to reproduce a "transosseous equivalent" anchor repair, but rather to consider a "true arthroscopic transosseous" technique in conjunction with anchor repair to achieve a maximized gold standard repair while practicing value based medicine and minimizing cost.

\section{Situation specific transosseous techniques}

All transosseous techniques offer several permutations and combinations of suture patterns. The simple repair, the box repair, and the $\mathrm{X}$ box repair, are demonstrated in Figure 2. These techniques allow for multiple small diameter fixation points to create whichever fixation pattern the surgeon chooses as appropriate.

A promising technique for small or partial tears (PASTA) is a single tunnel repair (Figure 3). As demonstrated, this repair can create excellent coaptation of the tendon, with added strength from suture grasping stitches. A single tunnel in small repair area can provide " 2 for 1 " fixation points relative to a single anchor with a true transosseous, circumferential stitches rather than a transosseous equivalent construct with multiple anchors. It is likely that lateral anchors in this clinical situation add little value relative to their cost.

For those new to tunneling techniques, a transitional approach with hybrid techniques may be of value, just as they have been with transitioning from open to mini-open to arthroscopic approaches for rotator cuff repair. Two hybrid techniques have been explored to provide maximum strength of repair in necessary situations at minimum cost, maximizing benefits of both repair strategies. There are an almost unlimited amount of anchor tunnel construct iterations and permutations possible, depending on specific needs. However, in the author's experience, two have proved to be particularly useful, especially as a surgeon transitions from experience in all anchor approaches and becoming familiar with suture management.

Anchor Bounded hybrid Repair (Figure 4): This technique prioritizes anchors as the primary means of fixation, and allows for the repair of any sized cuff tear with a transosseous equivalent four anchor construct. This repair is typically applied in severely retracted tears, reconstructions, or tears with noncompliant tissue. Anchors are placed at the margins of the tear, securing the mechanically important cables of the rotator cuff, while the intervening tissue is circumferentially opposed to bone with either one or two transosseous tunnels. The tunnels between the anchors respect the biology and bone stock of the tuberosity, while adding additional circumferential compression points of the tendon footprint. Additional tunnels may be created as needed for "dog ears" or further cuff reduction. This paradigm may also be used in superior capsular or graft augmentations, as there are often edges of the graft which must be secured for stability of the graft interface, even after multiple anchors have been used (Figure 5).

Tunnel bounded, minimal anchor hybrid repair (Figure 6): Tunnel bounded hybrid repair technique prioritizes tunnels as the primary fixation and allows for repair of any cuff tear with at least a single anchor and multiple tunnels using a reusable device, creating a high value repair with five fixation points. A double or triple anchor is placed at the center of the repair construct. Two tunnels are placed at the anterior and posterior margins of the tear. Three sutures are placed in each tunnel. After passing all sutures through the tendon, 
the anchor sutures are tied at the medial margin of the repair site. A passing suture is used to shuttle the medial anchor sutures through the lateral tunnels in the inferior to superior direction, reconstructing the footprint as in a transosseous equivalent repair, without the need for extra lateral anchors. These sutures are then tied back to themselves, creating a medial mattress component with fixation to the anchor. The remaining simple transosseous sutures are then tied, using the lateral suture limb as the post. Only one anchor is used in this construct at the point of medial stress concentration, while the footprint is restored using circumferential compression sutures without the need for more lateral anchors. As many other single tunnels may be added as necessary to augment the repair. The medial tails of the sutures may then by incorporated into a secondary backup lateral anchor if desired, creating a double anchor hybrid repair (Figure 7). Anchors and tunnels are thus used synergistically to maximize repair integrity and minimize cost.
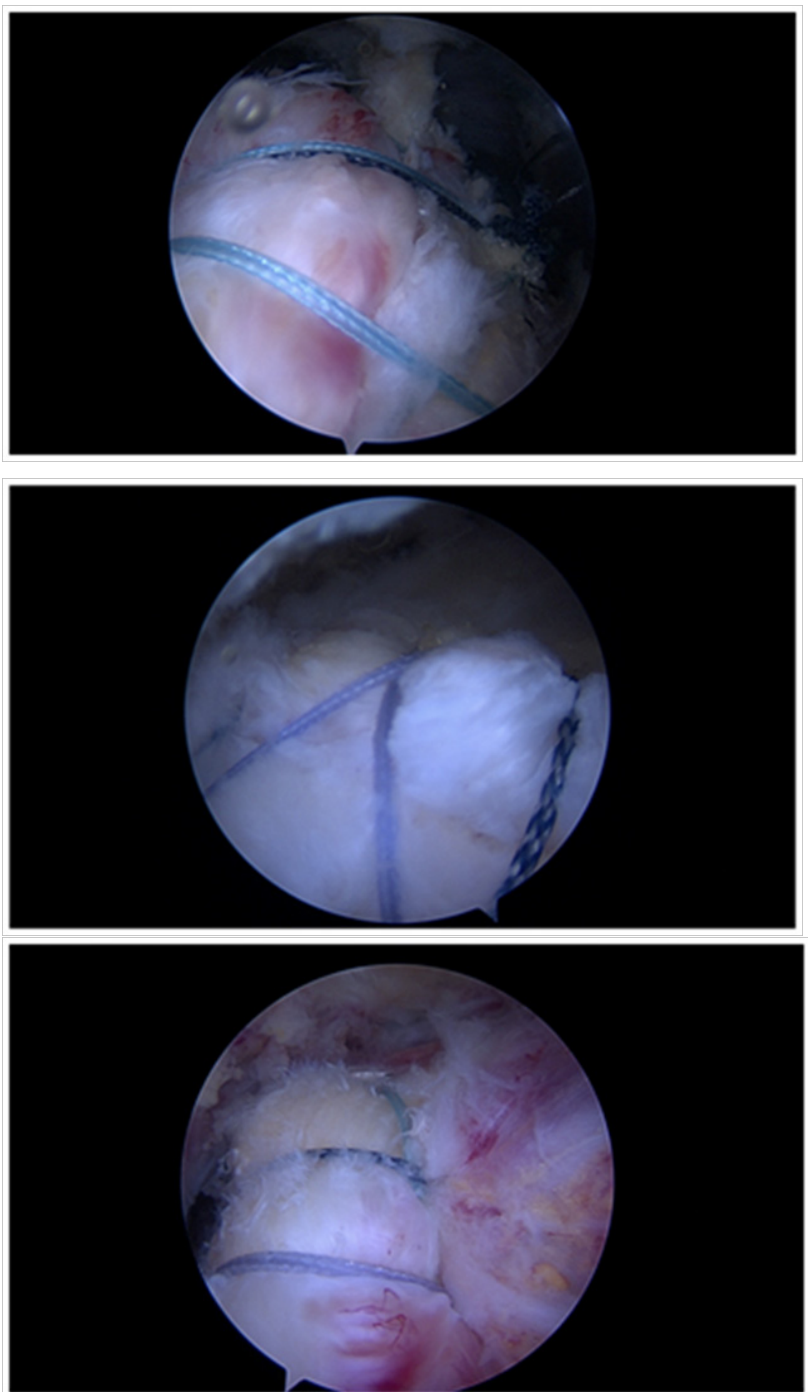

Figure 2 Various all transosseous repairs: $a$ ) simple $b$ ) $\mathrm{X}$ box c) three tunnel.

Biceps tenodesis (Figure 8): Arthroscopic supra pectoral approaches to biceps tenodesis that release the sheath are an option for biceps tenodesis, likely because pain relief may be provided by releasing the biceps sheath. ${ }^{24,31} \mathrm{~A}$ transosseous approach in this setting reduces anchor use, and obviates the use of a potentially troublesome wound in the axilla. Bone in this area is the most robust in the proximal humerus, and thus readily accessible for strong fixation in anchorless techniques.
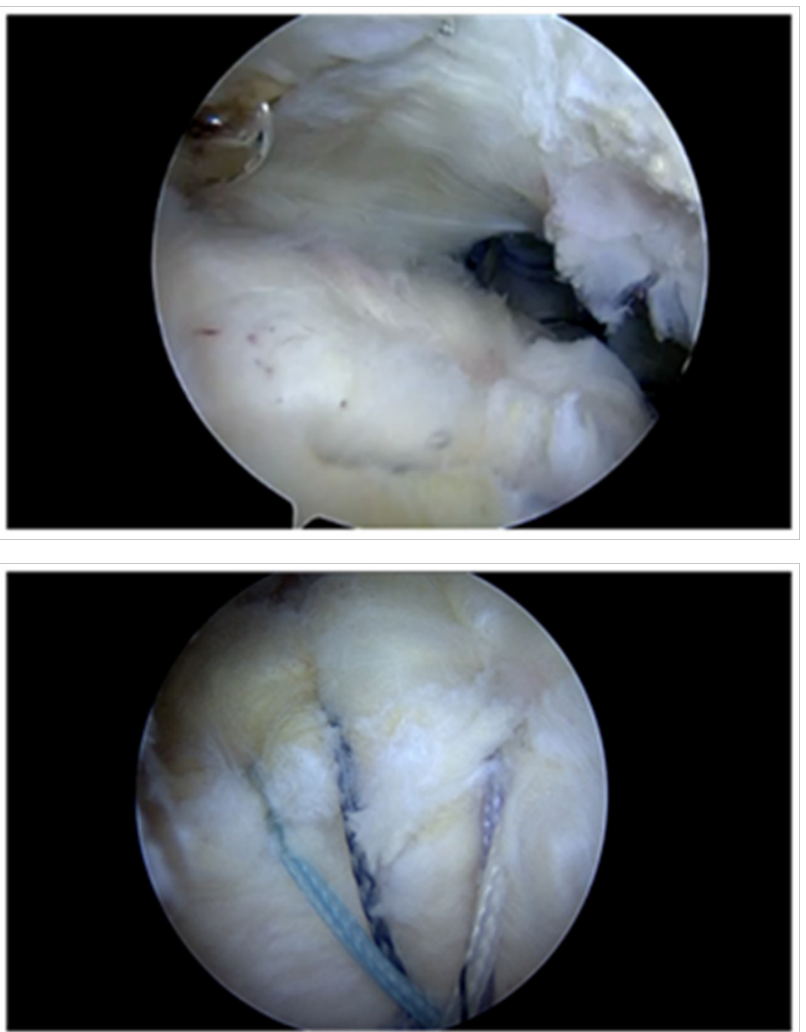

Figure 3 Single tunnel repair for a small rotator cuff tear.

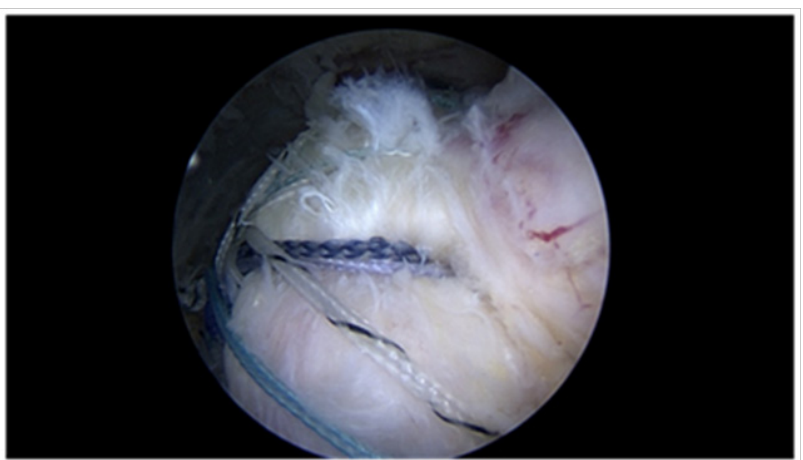

Figure 4 Anchor bounded hybrid repair.

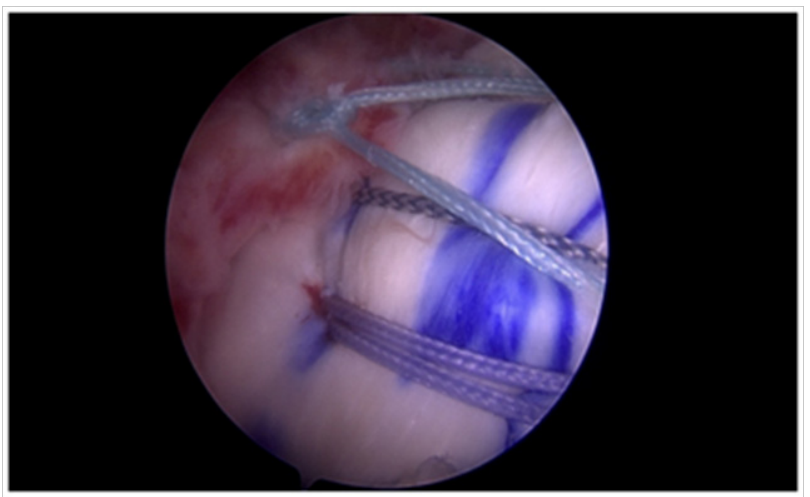

Figure 5 Superior capsular reconstruction with transosseous augmentation.

The biceps may be released, or initially left in situ for this technique. We prefer to release the biceps to allow for an interesting tendon grasping repair. Two tunnels are created with an awl from an anterolateral portal after releasing the biceps sheath. The first 
tunnel is created from the same portal in the inferior position, just at the superior margin of the pectorals major and above the anterior circumflex vessels. The doubled suture is retrieved through the tendon from an accessory inferomedial portal, and a locking loop is formed utilizing a retrograde passing device. This suture loop is then locked with the lateral suture tail and tied arthroscopcially with the biceps at physiological length from a posterior arthroscopic grasper. The second, more superior tunnel is then created using the arthroscopic tunneler. This double passing suture is then brought through the tendon using a retrograde passing device from the inferomedial portal. Grasping instruments are used to pass the loop over the tendon, creating a strong circumferential grasp on the tendon. The retrograde device is then used to create another locking loop through the circumferential loop, creating excellent fixation with a "cinch loop configuration". These sutures are then tied arthroscopically with the post lateral. The remaining tendon is removed, and the tendon ends are annealed with the electrocautery. ${ }^{32-38}$

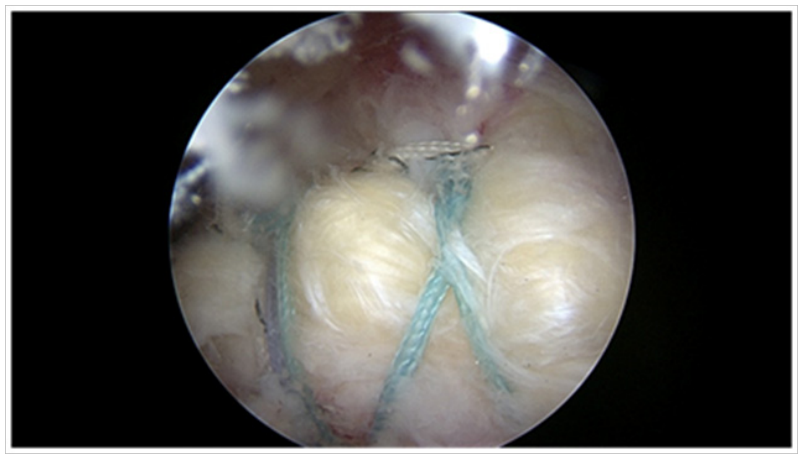

Figure 6 Single anchor: example of a tunnel bounded repair with a single anchor medially. Footprint reconstruction is achieved without the need for lateral anchors.
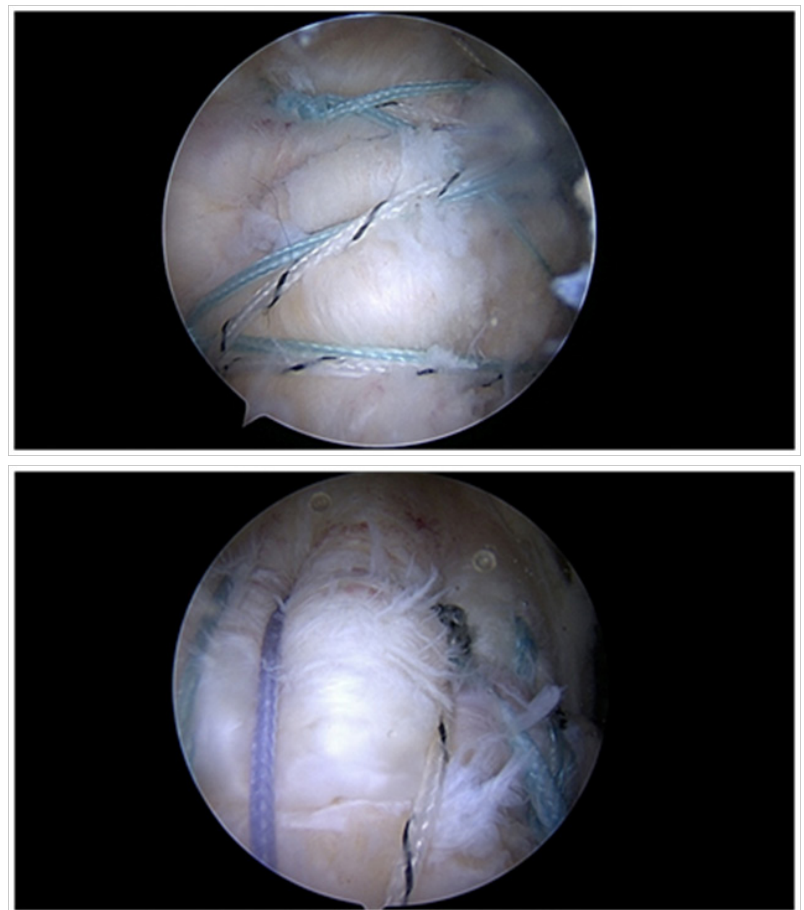

Figure 7 Tunnel bounded, double anchor repair: one medial and one lateral anchor are utilized with two tunnels, creating six fixation points.

Reusable arthroscopic transosseous tunneling is a reproducible and safe approach which adds multiple options to the arthroscopic surgeon's armamentarium for many commonly performed arthroscopic procedures. These approaches may offer another surgical option for soft tissue repair which respects biology, offers a biomechanically sound repair, creates "two for one fixation points" with circumferential tendon compression, and synergies well with existing technology. As clinician's incentives are increasingly aligned for value based treatments, these techniques could be employed to maximize clinical outcome, while minimizing cost in the value based era of medicine.

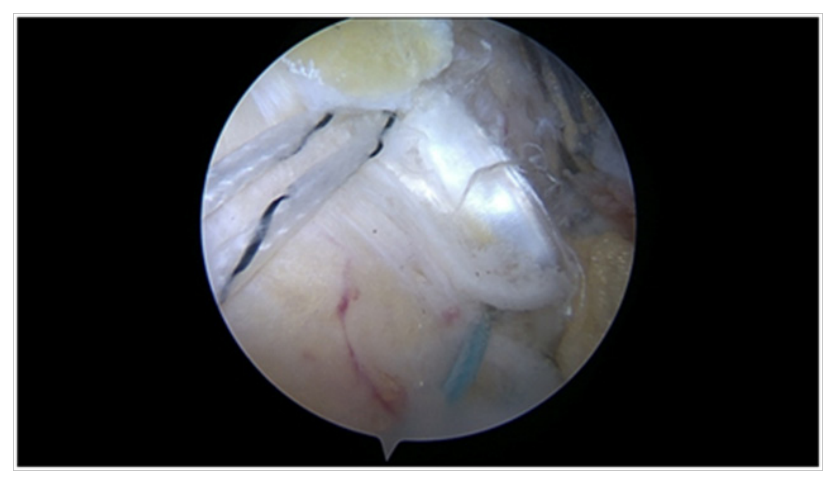

Figure 8 Biceps tenodesis: All transosseous suprapectoral biceps tenodesis with tendon grasping sutures.

\section{Acknowledgments}

None.

\section{Conflicts of interest}

None.

\section{References}

1. Black EM, Higgins LD, Warner JJP. Value Based Shoulder Surgery: Practicing Outcomes Driven, Cost Conscious Care. J Shoulder Elbow Surg. 2013;22(7):1000-1009.

2. Yu S, Zuckerman J. 5 Points on Orthopedics in US Healthcare. The Amercian Journal of Orthopedics. 2015;44(12):538-541.

3. Cordasco FA, McGinley BJ, Charlton T. Rotator cuff repair as an outpatient procedure. J Shoulder Elbow Surg. 2000;9(1):27-30.

4. Churchill RS, Ghorai JK. Total cost and operating room time comparison of rotator cuff repair techniques at low, intermediate, and high volume centers: mini-open versus all-arthroscopic. J Shoulder Elbow Surg. 2009;19(5):716-721.

5. Genuario JW, Donegan RP, Hamman D, et al. The cost-effectiveness of single-row compared with double-row arthroscopic rotator cuff repair. J Bone Joint Surg Am. 201294(15):1369-1377.

6. Srikumaran U, Hannan C, Kilcoyne K, et al. Arthroscopic Anchored Versus Transosseous Cuff Repair: A Comparison of Clinical Outcomes and Structural Integrity. AAOS presentation, Florida, USA. 2016.

7. Adla DN, Rowsell M, Pandey R. Cost-effectiveness of open versus arthroscopic rotator cuff repair. J Shoulder Elbow Surg. 2010;19(2):258261

8. Narvy S, Ahluwalia A, Vangsness CT. Analysis of Direct Cost of Outpatient Arthroscopic Rotator Cuff Repair. Am J Orthop. 2016;45(1):7-11.

9. Moosmayer S, Lund G, Seljom US, et al. Tendon repair compared with physiotherapy in the treatment of rotator cuff tears. A randomized controlled study in 103 cases with a five-year follow up. J Bone Joint Surg Am. 2014;96(18):1504-1514.

10. Payne A, Slover J, Inneh I, et al. Orthopaedic Implant waste:analysis and quantification. Am J Orthop. 2015;44(12):554-560. 
11. Russell RD, Knight JR, Mulligan E, et al. Structural integrity after rotator cuff repair does not correlate with patient function and pain: a meta-analysis. J Bone Joint Surg Am. 2014;96(4):265-271.

12. McLaughlin HL. Lesions of the musculotendinous cuff of the shoulder: the exposure and treatment of tears with retraction. 1944. J bone joint surg Am. 1944;304:3-9.

13. Ramsey ML, Getz CL, Parsons BO. What's new in shoulder and elbow surgery. J bone Joint surg Am. 2009;92(4):1047-1061.

14. Bishop J, Klepps S, Lo IK, et al. Cuff integirty after arthroscopic versus open rotator cuff repair: a prospective study. J shoulder elbow Surg. 2006;15(3):290-299.

15. McCallister WV, Parson IM, Titleman RM, et al. Open rotator cuff repai without acromioplasty. J Bone Joint Surg AM. 2005;87(6):1278-1283.

16. Cicak N, Klobucar H, Bicanic G, et al. Arthroscopic transosseous suture anchor technique for rotator cuff repairs. Arthroscopy. 2006;22(5):565.

17. Kim KC, Rhee KJ, Shin HD, et al. Arthroscopic transosseous rotator cuff repair. Orthopeidcs. 2008;31(4):327-330.

18. Krishnan SG. Arthroscopic trans-osseous rotator cuff repair: going back to the future. Presentation at San Diego Shoulder meeting, California, USA. 2009.

19. Garofalo R, Castagna A, Borroni M, et al. Arthroscopic transosseous (anchorless) rotator cuff repair. Knee Surg Sports Traumatol Arthrosc. 2011;20(6):1031-1035.

20. Burkhart SS, Nassar J, Schenck RC, et al. Clinical and anatomic considerations in the use of a new anterior inferior submaxillary nerve portal. Arthrsocopy. 1996;12(5):634-637.

21. Srikumaran U, Romine L, Hannan C, et al. Arthroscopic transosseous rotator cuff repair is cost effective compared to anchored technique. Poster presentation at San Diego Shoulder meeting, California, USA. 2013.

22. Kuroda S, Ishige N, Mikasa M. Advantages of arthroscopic transosseous suture repair of the rotator cuff without the use of anchors. Colin Ortho Relat Res. 2013;471(11):3514-3522.

23. Black EM, Linn A, Skrikumaran U, et al. Arthroscopic Transosseous Rotator Cuff repair: technical note, outcomes, and complications. Orthopaedics. 2015;38(5):352-358.

24. Kahlenberg CA, Patel RM, Nair R, et al. Clinical and Biomechanical Evaluation of an All-arthroscopic Suprapectoral Biceps Tenodesis. Orthop J Sports Med. 2014;2(10):2325967114553558.

25. Garrigues GE, Lazarus MD. Arthroscopic bone tunnel augmentation for rotator cuff repair. Orthopaedics. 2012;35(5):392-397.
26. Fox MP, Auffarth A, Tauber M, et al. A novel transosseous button technique for rotator cuff repair. Arthroscopy. 200824(9):1074-1077.

27. Nho SJ, Michael KS, Seth LS, et al. Systematic review of arthroscopic rotattor cuff repair and mini-open rotator cuff repair. J Bone Joint Surg Am. 2007;89:127-136

28. Behrens SB, Bruce B, Zonno AJ, et al. Initial fixation strength of transosseous equivalent suture bridge repair is comparable with transosseous repair. Am J Sports Med. 2012;40(1):133-140.

29. Werner BC, Evans CL, Holzgrefe RE, et al. Arthroscopic suprapectoral and open sub pectoral biceps tenodesis: a comparison of minimum 2 year clinical outcomes. Am J Sports Med. 2014;42(11):2583-9580.

30. Vitale MA, Vitale MG, Zivin JG, et al. Rotator Cuff Repair: an analysis of utility scores and cost-effectiveness. J Shoulder Elbow Surg. 2007;16(2):181-187.

31. Tauber M, Koller H, Resch H. Transosseous arthroscopic repair of partial articular-surface supraspinatus tendon tears. Knee Surg Sports Traumatol Arthrosc. 2008;16(6):608-613.

32. Kummer FJ, Hahn M, Day M, et al. Laboratory comparison of a new arthroscopic transosseous rotator cuff repair technique to a double row transosseous equivalent repair using anchors. Bull Hosp Jt Dis. 2013;71(2):128-131.

33. Bisson LJ, Manohar LM. A biomechanical comparison of transosseoussuture anchor and suture bridge rotator cuff repairs in cadavers. Am J Sports Med. 2009;37(10):1991-1995.

34. Johst PW, Khair MM, Chen DX, et al. Suture number determines strength of rotator cuff repair. J Bone Joint Surg Am. 2012;94(14):100.

35. Jo CH, SHin JS, Park IW, et al. Multiple channeling improves the structural integrity of rotator cuff repair. Am J Sports Med. 2013;41(11):2650-2657.

36. Milano G, Saccomanno MF, Careri S, et al. Efficacy of marrow stimulating technique in rotator cuff repair: a prospective randomized study. Arthroscopy. 2011;29(5):802-810.

37. Cho NS, Yi JW, Lee BG, et al. Retear patterns after arthroscopic rotator cuff repair: Single-row versus suture bridge technique. Am J Sports Med. 2010;38(4):664-671

38. Sanders B, Lavery KP, Pennington S, et al. Clinical success of biceps tenodesis with and without release of the transverse humeral ligament. $J$ Shoulder Elbow Surg. 2012;21(1):66-71. 